\title{
Successful Surgical Intervention of Strangulated Ileus with a Simple Cut of the External Iliac Vein without Vein Reconstruction
}

\author{
Takanori Hishikawa $^{a}$ Shoji Oura ${ }^{b}$ Masafumi Tomita ${ }^{a}$ \\ aDepartment of Surgery, Kobe Tokushukai Hospital, Kobe, Japan; b Department of Surgery, \\ Kishiwada Tokushukai Hospital, Kishiwada, Japan
}

\section{Keywords}

External iliac vein · Internal hernia $\cdot$ Strangulated ileus · Thrombi in the femoral vein

\begin{abstract}
A 67-year-old woman with epigastralgia was referred to our hospital. The patient had undergone hysterectomy, bilateral oophorectomy, omentectomy, and radical pelvic and para-aortic lymph node dissection for her ovarian cancer 6 years before. Despite the gastrointestinal decompression therapy under the presumed diagnosis of adhesive ileus, computed tomography scans taken 3 days after the onset of epigastralgia showed marked dilatation of the small intestine and an oval high-density mass, that is, thrombi, in the right femoral vein. Aggravation of ileus with the thrombi in the femoral vein made us to treat the patient with surgery. Intraoperative findings showed that the terminal ileum was strangulated by a gap between the exposed right external iliac vein and artery presumably formed by pelvic lymph node dissection. Distal ileum strangulated by the gap, however, showed no ischemic change with no surgically available peritoneum left around the external iliac vein. To prevent the pulmonary embolism and the recurrence of this type of ileus due to both the thrombi and the persistent gap, we released the strangulated ileum with a simple cut of the external iliac vein without vein reconstruction. The patient recovered uneventfully and was discharged on the 13th day after operation. The patient has been well with nominal right leg edema. In this situation, that is, internal hernia caused by external iliac vessels with thrombi in the femoral vein and no leg edema, a simple cut of the external iliac vein without vein reconstruction is a feasible treatment option.
\end{abstract}




\section{Introduction}

Small bowel obstruction occurs when the normal flow of the intestinal contents is interrupted. Etiologies of small bowel obstruction mainly consist of intraperitoneal adhesions, tumors, hernias, Crohn's disease, gall stones, volvulus, and intussusception in descending order of frequency [1-4]. Of these, postoperative adhesions account for approximately $60 \%$ of the causes of small bowel obstruction.

Decision-making for surgical intervention is the most important judgment for attending physicians to treat small bowel obstruction on the one hand. Whether to resect or to preserve the affected small intestine is the crucial judgment for surgeons when the small intestine shows some ischemic change on the other hand. In addition, how to manage the vessels, when causing small intestinal obstruction, is another important issue, though much less common than the ischemic event, for surgeons.

With the development of medical science, various cancers have come to be detected at an early stage, leading to the prolonged survival of cancer patients. An increase in number of cancer survivors directly leads to an increase in patients who develop postoperative ileus. Physicians, therefore, have a significantly increased opportunity to properly diagnose and treat the patients with various types of ileus now. We herein report an extremely rare case of strangulated ileus due to external iliac vessels after pelvic lymph node dissection successfully treated with laparoscopic release of the strangulated ileum with a simple cut of the external iliac vein without vein reconstruction.

\section{Case Report}

A 67-year-old woman was referred to our hospital due to sudden onset of epigastralgia after dinner. The patient had undergone hysterectomy, bilateral oophorectomy, omentectomy, and pelvic and para-aortic lymph node dissection for her ovarian cancer 6 years before. Computed tomography (CT) on admission showed no dilatation of the small intestine. The patient was afebrile and had flat and soft abdomen with tenderness from the upper abdomen to the right lower abdomen. Blood gas analysis did not suggest intestinal necrosis, that is, neither the elevation of serum lactate level nor the metabolic acidosis. The patient, therefore, initially received gastrointestinal decompression and fluid therapy under the presumed diagnosis of adhesive ileus, unfortunately leading to the failure of symptom relief and the aggravation of ileus on follow-up CT (Fig. 1a). We, therefore, performed laparoscopic surgery to the patient on the 3rd day after admission. On laparoscopic inspection, we found minimum intra-abdominal adhesions, yellowish serous ascites without cancer cell dissemination, marked dilatation of the ileum, part of the terminal ileum passing through under the right external iliac vein without ischemic change, and no surgically available peritoneum left around the right external iliac vein (Fig. 2a). In addition, we had preoperatively found presumed thrombi in the right external iliac and femoral veins on nonenhanced CT (Fig. 1b) but no clinically significant swelling in the right leg. We, therefore, cut the right external iliac vein without vein reconstruction using an endoscopic stapler for the release of the strangulated ileum to prevent both the pulmonary embolism and the recurrence of this type of ileus (Fig. 2b). The patient recovered uneventfully with marked relief of the uncomfortable symptoms due to ileus. Ultrasonography on the 7 th postoperative day showed thrombi extending from the distal external iliac vein to the femoral vein without further extension beyond the femoral vein. The patient began to wear an elastic stocking on the right leg during the admission period and was discharged on the 13th postoperative day. The patient has been doing well with

\section{Karger'}



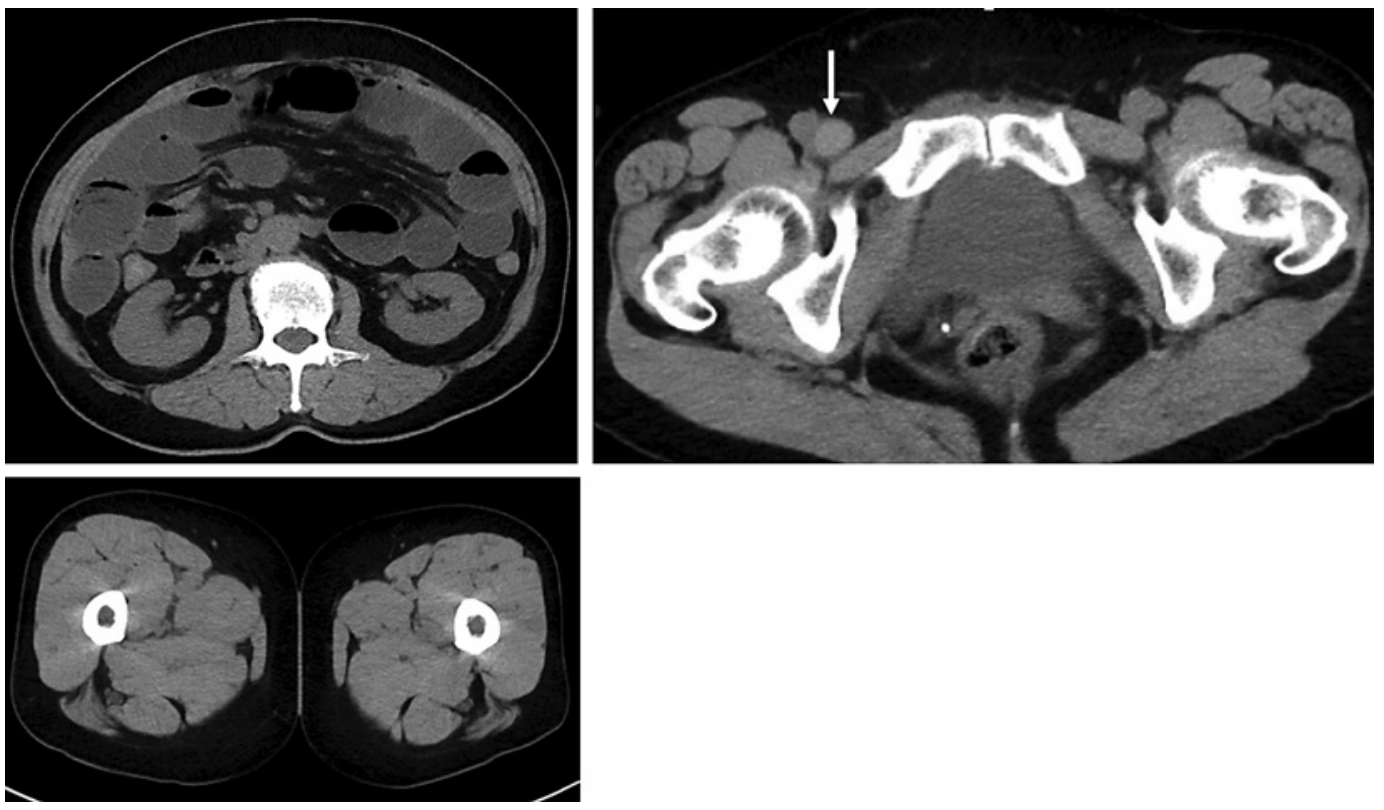

Fig. 1. CT of the abdomen and legs. a CT showed dilatation of the small intestine and fluid retention in the intestine. b CT showed an oval and homogenous mass, that is, presumed thrombi (arrow), in the femoral vein. c CT after discharge showed neither skin edema nor venous dilatation in the right leg. CT, computed tomography.
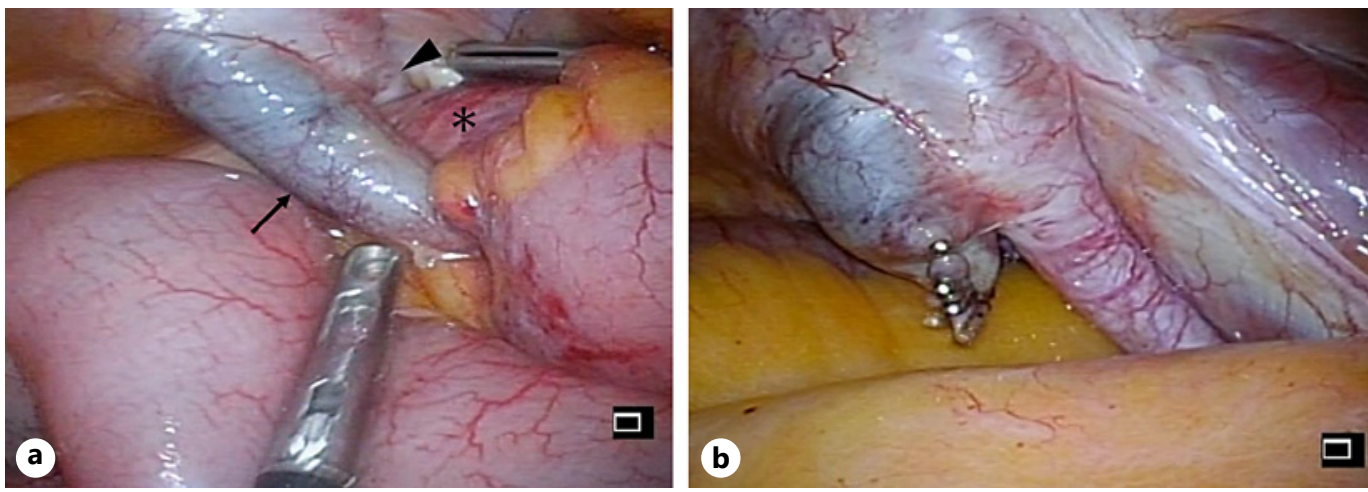

Fig. 2. Intraoperative findings. a Terminal ileum (asterisk) strangulated between the external iliac vein (arrow) and artery (arrowhead) was cut with an endoscopic stapler. b Strangulated terminal ileum was released. External iliac artery and the stump of the external iliac vein were observed.

no walking disturbance, no daily life impairment, and nominal lower limb swelling (Fig. 1c, 3).

\section{Discussion}

Small bowel obstruction can be mechanical and functional [1]. The former is a condition in which the intestinal contents cannot pass through intestinal lumen due to narrowing or obstruction caused by some kind of etiologies in or outside the intestine, while the latter is a similar condition without apparent narrowing or obstruction of the intestinal lumen. In this 
Fig. 3. Photo of the lower legs 2 weeks after discharge. Nominal swelling of the right lower leg was observed.

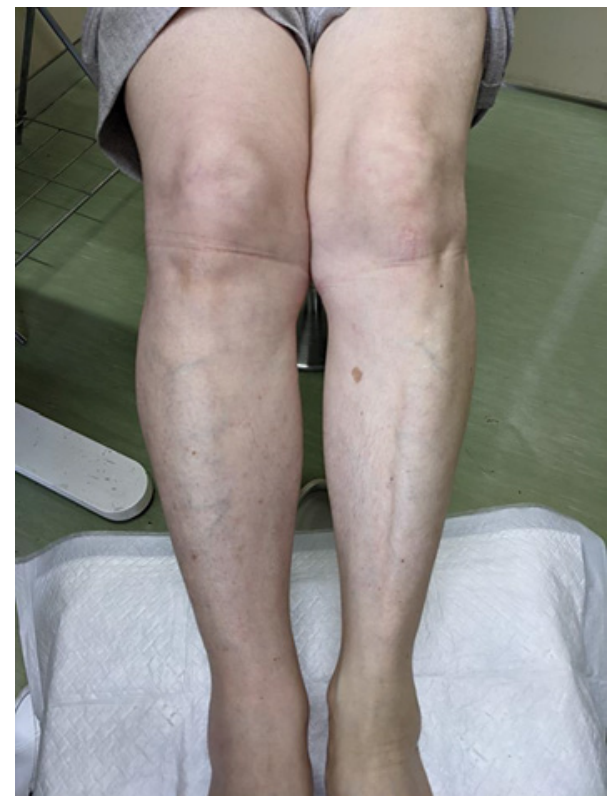

case, ileus was caused by the passing of the terminal ileum through the gap generated between the external iliac artery and vein, that is, internal hernia, presumably caused by lymph node dissection for her ovarian cancer. In general, surgical manipulation brings some adhesion to the tissues or organs in the operated field, generally leading to the difficult reoperation to the same field. We cannot speculate why this patient did not develop adhesion around the external iliac vessels. If adhesion had been present, it should have never caused this type of strangulated ileus.

Abdominal CT plays the most important role in the diagnosis of ileus. Abdominal CT can not only identify the site that causes ileus but also predict the pathophysiology of the ileus with the symbolic signs such as closed loop sign [5], beak sign [6], whirl sign [7], and dirty fat sign [8]. Contrast-enhanced CT can further depict the ischemic changes of the affected intestine. The presence of comorbid disease of asthma in this patient forced us to evaluate the ileus not with contrast-enhanced CT but with nonenhanced CT, leading to the insufficient preoperative diagnosis. Contrast-enhanced CT, if having been performed, should have enabled us to make more precise preoperative diagnosis such as extent of thrombi and the correlation between the strangulation point and the pelvic vessels. Accurate image diagnosis should have led us to another therapeutic option requiring more advanced surgical expertise, that is, external iliac vein reconstruction after removing the thrombi in the external iliac and femoral veins.

It was very difficult for physicians including us to predict whether surgically available peritoneum existed or not around the external iliac vein on any images. Vein reconstruction, if having been done, should have led us further to the annoyance how to cover the gap between the external iliac vessels. Although abdominal CT showed presumed massive thrombi in the femoral vein, no leg edema was observed in this patient. We, therefore, selected a simple cut of the external iliac vein to resolve the strangulation ileus and to prevent both the pulmonary embolism and the recurrence of this type of ileus, leading to no aggravation of the femoral thrombi. Surgeons generally try to preserve large vessels as much as possible especially on emergent or urgent operation. Oliver et al. [9], however, reported that ligating the common or external iliac veins was associated with a low incidence of prolonged leg swelling in patients with injury to the iliac veins. In addition, there is no evidence of increased leg edema in

\section{Karger'}


patients treated with a vein cut alone compared with those treated with a vein cut followed by vein reconstruction. In fact, postoperative CT and photos clearly showed nominal swelling of the patient's leg. Naturally, the patient developed no abnormalities in walking and has lived a normal daily life.

Surgery to the abdominal malignancies generally consists of resection of primary malignancy and regional node dissection, leading to the isolation of vessels, ureters, and even nerves. All these structures can be candidates for the etiologies for strangulation ileus by internal hernia. Surgical oncologists, therefore, should try to preserve the peritoneum in the primary pelvic cancer operation whenever possible [10]. In conclusion, we experienced a case of strangulation ileus in which the small intestine strayed into the gap caused by the exposed external iliac vessels after pelvic lymph node dissection for gynecologic cancer and was successfully released with a simple cut of the external iliac vein without vein reconstruction.

\section{Statement of Ethics}

Written informed consent was obtained from the patient for publication of this case report and any accompanying images. Institutional Review Boards (IRB) of Kobe and Kishiwada Tokushukai Hospital do not require IRB review for a single case report.

\section{Conflict of Interest Statement}

The authors declare that they have no conflicts of interest.

\section{Funding Sources}

There were no funding sources.

\section{Author Contributions}

T. Hishikawa contributed to the design of the report. S. Oura drafted the manuscript. M. Tomita treated the patient. All authors have read and approved the final version of the manuscript.

\section{Data Availability Statement}

All data generated during this study are included in this article. Further inquiries can be directed to the corresponding author.

\section{References}

1 Miller G, Boman J, Shrier I, Gordon PH. Etiology of small bowel obstruction. Am J Surg. 2000;180:33-6.

2 Rami Reddy SR, Cappell MS. A systematic review of the clinical presentation, diagnosis, and treatment of small bowel obstruction. Curr Gastroenterol Rep. 2017;19(6):28.

3 Bizer LS, Liebling RW, Delany HM, Gliedman ML. Small bowel obstruction: the role of nonoperative treatment in simple intestinal obstruction and predictive criteria for strangulation obstruction. Surgery. 1981;89:407-13.

\section{Karger's}


4 Ten Broek RPG, Krielen P, Di Saverio S, Coccolini F, Biffl WL, Ansaloni L, et al. Bologna guidelines for diagnosis and management of adhesive small bowel obstruction (ASBO): 2017 update of the evidence-based guidelines from the world society of emergency surgery ASBO working group. World J Emerg Surg. 2018;13:24.

5 Balthazar EJ, Birnbaum BA, Megibow AJ, Gordon RB, Whelan CA, Hulnick DH. Closed-loop and strangulating intestinal obstruction: CT signs. Radiology. 1992;185:769-75.

6 Metcalfe AM, DiSantis DJ, Alexander LF. The “double beak sign” on CT. Abdom Radiol. 2021;46(4):1771-2.

7 Khurana B. The whirl sign. Radiology. 2003;226:69-70.

8 Toriihara A, Yamaga E, Nakadate M, Oyama J, Tateishi U. Detection of unexpected emergency diseases using FDG-PET/CT in oncology patients. Jpn J Radiol. 2017;35:539-45.

9 Oliver JC, Bekker W, Edu S, Nicol AJ, Navsaria PH. A ten year review of civilian iliac vessel injuries from a single trauma centre. Eur J Vasc Endovasc Surg. 2012;44:199-202.

10 Franchi M, Ghezzi F, Zanaboni F, Scarabelli C, Beretta P, Donadello N. Nonclosure of peritoneum at radical abdominal hysterectomy and pelvic node dissection: a randomized study. Obstet Gynecol. 1997;90:622-7. 